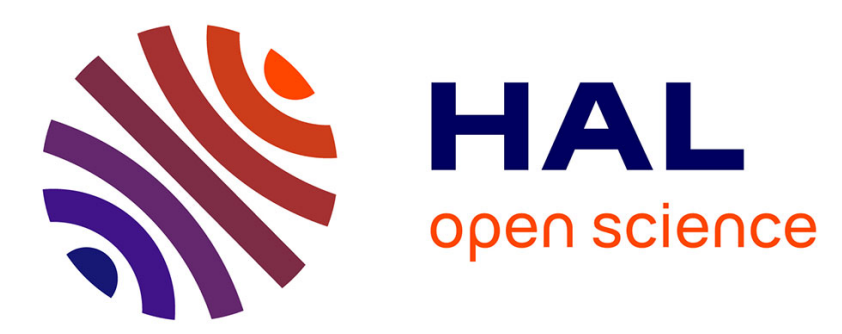

\title{
Simulation of instabilities in thin nanostructures by a perturbation approach
}

\author{
Y. Cong, Julien Yvonnet, H. Zahrouni
}

\section{To cite this version:}

Y. Cong, Julien Yvonnet, H. Zahrouni. Simulation of instabilities in thin nanostructures by a perturbation approach. Computational Mechanics, 2014, 53 (4), pp.739-750. 10.1007/s00466-013-0927-7 . hal-00958886

\section{HAL Id: hal-00958886 https://hal.science/hal-00958886}

Submitted on 8 Sep 2014

HAL is a multi-disciplinary open access archive for the deposit and dissemination of scientific research documents, whether they are published or not. The documents may come from teaching and research institutions in France or abroad, or from public or private research centers.
L'archive ouverte pluridisciplinaire HAL, est destinée au dépôt et à la diffusion de documents scientifiques de niveau recherche, publiés ou non, émanant des établissements d'enseignement et de recherche français ou étrangers, des laboratoires publics ou privés. 


\title{
Simulation of instabilities in thin nanostructures by a perturbation approach
}

\author{
Y. Cong · J. Yvonnet · H. Zahrouni
}

Received: date / Accepted: date

\begin{abstract}
A new numerical method is proposed to simulate instabilities in thin atomistic structures in quasi-static regime. In contrast with previous approaches based on energy minimization or Newton-Raphson methods, the present technique uses a series expansion of atomistic displacements with respect to a loading path parameter, truncated at high orders. The nonlinear set of equations defined by minimizing the potential energy of the discrete system with respect to nuclei positions is then transformed into a sequence of linear sets of equations, which can be solved efficiently. The solution can be described along very large loading steps without correction, resulting in a significant reduction of matrices to be inverted. Finally, the treatment of limit points and snap-back/snap-through arising when instabilities occur is simplified due to a continuous description with respect to the loading path parameter. The method is applied to the analysis of single carbon atom layers nanostructures like graphene sheets or nanotubes in traction or compression regimes. Accuracy and efficiency of the technique is demonstrated by comparisons with iterative Newton procedures.
\end{abstract}

Y. Cong

Université de Lorraine, Laboratoire d'Étude des Microstructures et de Mécanique des Matériaux (LEM3), UMR CNRS 7239, Ile du Saulcy, 57045 Metz, France

Tel.: $+33-387315358$

Fax: +33-387315366

E-mail: yu.cong@univ-lorraine.fr

J. Yvonnet

Université Paris-Est, Laboratoire Modélisation et Simulation Multi-Échelle (MSME), UMR CNRS 8208, 5 Boulevard Descartes, 77454 Marne-la-Vallée, France

Tel.: +33-160957795

Fax: +33-160957799

E-mail: julien.yvonnet@univ-paris-est.fr

H. Zahrouni

Université de Lorraine, Laboratoire d'Étude des Microstructures et de Mécanique des Matériaux (LEM3), UMR CNRS 7239, Ile du Saulcy, 57045 Metz, France

Tel.: $+33-387315424$

Fax: +33-387315366

E-mail: hamid.zahrouni@univ-lorraine.fr 
Keywords Asymptotic numerical method - Molecular statics · Graphene · Nanotubes $\cdot$ Instabilities

\section{Introduction}

Mechanics of thin nanostructures has recently attracted a tremendous attention due to potential applications of graphene, nanotubes or other low-dimensional nano objects in integrated electromechanical miniaturized systems. When dealing with thin nanostructures, instabilities such as buckling [31,15,45] and wrinkling $[41,39,12]$ may occur even under moderate compressive loading conditions. It was shown through simulations that buckling and wrinkles can affect mechanical properties of thin objects $[49,4]$. This motivated many theoretical and experimental studies on the topic of instabilities in nanostructures. Modeling methods based on continuum methods have been proposed to deal with thin nanostructures, employing e.g. shell or beam theories. An early contribution was proposed by Yakobson [44], who analyzed the buckling of single-walled nanotubes using a thin elastic shell model. A series of contributions was proposed by $\mathrm{Ru}$ et al $[35,36,37,38,42]$ who extended the approach to deal with multi-walled carbon nanotubes, for which van der Waals forces were introduced to model interaction between layers. Other classical continuum theories were employed by several authors, like Govindjee et al [16], who modeled multi-walled nanotubes using a beam model.

However, It has been recognized that classical continuum methods do not naturally include local effects due to the discrete atomistic structure [26] and that atomistic methods are required to fully understand mechanisms at the nanoscale, or to identity parameters for higher scales continuum models. In [31], buckling of double graphene layers in compression interacting with van der Waals forces have been studied by a theoretical model, validated by Molecular Dynamics (MD) simulations. In [9], Chandra et al. investigated in-plane instabilities of bilayer graphene using atomistic finite element approaches to determine the overall properties of the sheet. In [17], instability patterns in nanomembranes containing gold nanoparticles were observed under compressive stress by means of MD simulations. In [26], Neek-Amal and Peeters examined instabilities occurring in circular monoloayer graphene subjected to radial load using MD. In [49] Zheng et al. looked into modification of mechanical properties and critical wrinkling strain in graphene sheets with respect to chemical functionalization. In [4] Arroyo and Belytschko studied the effects of wrinkling modes on the effective bending stiffness of multiwall carbon nanotubes. Wanga et al. [43] developed a hybrid-continuum mechanical and molecular mechanics framework to predict the compressive buckling strain of armchair and zigzag carbone nanotubes.

When dealing with simulation of quasi static loadings, several difficulties are induced. First, when MD [32] methods are employed, an artificial strain rate must be introduced, which can perturb the original problem. To avoid this issue, large simulation durations must be employed. Stable time steps in MD are very small (of the order of $10^{-15} \mathrm{~s}$ ), and can lead to extremely high computational costs. For instance, $\mathrm{Lu}$ and Bhattacharya [21] investigated the extension of a defected $(6,6)$ single-walled nanotube of $42 \AA$ in length under tensile loading. Numerical experiments showed that nearly 1.2 million time steps were needed to obtain a global length variation of $4.2 \AA$. In addition, several tests must be performed to ana- 
lyze the effects of different loading rates, which further multiplies computational efforts.

Another option is to consider quasi-statics (QS) molecular simulations, by directly seeking the equilibrium positions of atoms under the action of external load and nonlinear interatomic forces deriving from potentials. To solve the nonlinear set of equations, conjugate gradients (CG) [13,14], or Newton-Raphson (NR) methods can be used. Both represents respective drawbacks: on one hand, CG only requires computing the matrix of potential first derivatives, but its convergence is slow. On the other hand, Newton-like methods require computing the second derivatives but converge much faster near local minima of potential energy. However, NR solvers face severe lack of robustness when dealing with instabilities in general, and especially in the case of nanostructures, where local motion of atoms can induce limit points and negative definite tangent matrix.

In the present work, we propose a new simulation approach for dealing with quasi-static simulation of atomistic thin structures with instabilities. This technique extends a perturbation approach, called in the literature Asymptotic $\mathrm{Nu}$ merical Method (ANM) (see e.g. [48,25,47,46,1,19,6,7]), to molecular mechanics. ANM is a high order perturbation method which allows solving a set of nonlinear equations in an efficient manner. The displacement solution is expressed by a series expansion with respect to a loading path parameter, and truncated at high orders. As a consequence, the set of nonlinear equations is transformed into a sequence of linear equations which can be solved numerically. Approximations of the solution along large portions of the loading path are obtained continuously without iterations, and the treatment of limit points is simplified. These features motivate the choice of ANM for simulations of instabilities. The method has been recently applied to analyze multiscale instabilities in composite materials [27,29].

The outline of the paper is as follows: in section 2 , the equations of the atomistic quasi-static problem are formulated. In section 3, the Asymptotic Numerical Method is briefly recalled and its extensions to atomistic simulation are introduced in section 4. Finally, section 5 presents several numerical examples which are selected to demonstrate the efficiency and robustness of the present approach.

\section{Computational model for atomistic simulation}

\subsection{Problem formulation}

An isolated system of atoms or molecules is considered. The total energy is the sum of the kinetic and potential energies of the molecules. It is constant in time and identified as the Hamiltonian $H$, given by

$$
\begin{gathered}
H\left(\mathbf{x}_{1}(t), \mathbf{x}_{2}(t), \ldots, \mathbf{p}_{1}(t), \mathbf{p}_{2}(t), \ldots\right) \\
=\sum_{i} \frac{1}{2 m_{i}} \mathbf{p}_{i} \cdot \mathbf{p}_{i}+E_{\text {total }}\left(\mathbf{x}_{1}(t), \mathbf{x}_{2}(t), \ldots\right),
\end{gathered}
$$

where $m_{i}$ and $\mathbf{x}_{i}$ denote mass and position of atom $i$, respectively, $\mathbf{x}_{i}=\mathbf{X}_{i}+\mathbf{d}_{i}$, $\mathbf{X}_{i}$ is the original position of atom $i, \mathbf{d}_{i}$ the displacement of atom $i$, and $\mathbf{p}_{i}$ is the momentum defined by 


$$
\mathbf{p}_{i}=m_{i} \dot{\mathbf{x}}_{i}=m_{i} \dot{\mathbf{d}}_{i}
$$

In Eq. (2) the dot (.) indicates derivative with respect to time and $E_{\text {total }}$ is the potential energy defined as the sum of the energies due to any force fields, such as pair-wise interaction of the atoms, three-body potentials or others. It can be written as

$$
E_{\text {total }}\left(\mathbf{x}_{1}, \mathbf{x}_{2}, \ldots\right)=\sum_{i} E_{1}\left(\mathbf{x}_{i}\right)+\sum_{i, j>i} E_{2}\left(\mathbf{x}_{i}, \mathbf{x}_{j}\right)+\sum_{i, j>i, k>j} E_{3}\left(\mathbf{x}_{i}, \mathbf{x}_{j}, \mathbf{x}_{k}\right)+\ldots
$$

In the context of quasi-static simulations, the kinetic energy and time dependence are neglected in Eq. (1). Then the Hamiltonian reduces to the potential energy and the solution of the problem is found by

$$
\left\{\mathbf{x}_{1}, \mathbf{x}_{2}, \ldots\right\}=\operatorname{Argmin} E_{\text {total }}\left(\mathbf{x}_{1}, \mathbf{x}_{2}, \ldots\right) .
$$

In the present work, investigated systems consist in single carbon atoms layers, found in graphene or nanotubes. We adopt the modified Morse potential (see e.g. et al [5]). Compared to the conventional Morse potential [24], an additional energy contribution due to bond angle variation has been added to stabilize the numerical model of nanostructures, for which total internal energy is computed by summing up potentials evaluated at all atomic bonds. In that case we have

$$
E_{\text {internal }}=\sum_{i, j \in \Gamma_{1}} E_{\text {stretch }}\left(\mathbf{x}_{i}, \mathbf{x}_{j}\right)+\sum_{i, j, k \in \Gamma_{2}} E_{\text {angle }}\left(\mathbf{x}_{i}, \mathbf{x}_{j}, \mathbf{x}_{k}\right)
$$

where $\Gamma_{1}$ and $\Gamma_{2}$ are the set of indices related to pair and angular bonds, respectively. Introducing an external force $\mathbf{F}_{\alpha}$ applied to the atom $\alpha$, the total energy can be written as

$$
E_{\text {total }}=\left(\sum_{i, j \in \Gamma_{1}} E_{\text {stretch }}\left(\mathbf{x}_{i}, \mathbf{x}_{j}\right)+\sum_{i, j, k \in \Gamma_{2}} E_{\text {angle }}\left(\mathbf{x}_{i}, \mathbf{x}_{j}, \mathbf{x}_{k}\right)\right)-\lambda \sum_{\alpha} \mathbf{F}_{\alpha} \cdot \mathbf{x}_{\alpha}
$$

in which $\lambda$ is a loading amplitude parameter. Applying (4) gives the following set of nonlinear equations:

$$
\begin{gathered}
\frac{\partial E_{\text {total }}}{\partial \mathbf{x}_{m}}=\sum_{i, j \in \Gamma_{1}}\left[\mathbf{F}_{i-j}^{s}\right]_{m} \\
+\sum_{(i, j, k) \in \Gamma_{2}}\left[\mathbf{F}_{i-j-k}^{a}\right]_{m}-\lambda \mathbf{F}_{m}=\mathbf{0}, \quad k=1,2, \ldots, N_{a t},
\end{gathered}
$$

which have to be solved numerically, $N_{a t}$ being the total number of atoms. In Eq. (7), $\left[\mathbf{F}_{i-j}^{s}\right]_{m}$ and $\left[\mathbf{F}_{i-j-k}^{a}\right]_{m}$ are the forces created by the stretching and bending of the pair $(i-j)$ and angular bounds $(i-j-k)$ acting on the atom $m$, respectively, identified as the derivative of the two corresponding potentials:

$$
\left[\mathbf{F}_{i-j}^{s}\right]_{k}=\frac{\partial}{\partial \mathbf{x}_{k}}\left(E_{\text {stretch }}\left(\mathbf{x}_{i}, \mathbf{x}_{j}\right)\right),
$$


and

$$
\begin{aligned}
{\left[\mathbf{F}_{i-j-k}^{a}\right]_{l} } & =\frac{\partial}{\partial \mathbf{x}_{l}}\left(E_{\text {angle }}\left(\mathbf{x}_{i}, \mathbf{x}_{j}, \mathbf{x}_{k}\right)\right) \\
{\left[\mathbf{F}_{j-i-k}^{a}\right]_{l} } & =\frac{\partial}{\partial \mathbf{x}_{l}}\left(E_{\text {angle }}\left(\mathbf{x}_{j}, \mathbf{x}_{i}, \mathbf{x}_{k}\right)\right)
\end{aligned}
$$

where equations (9) and (10) correspond to the cases described in figure 1 (a) and 1 (b), respectively.

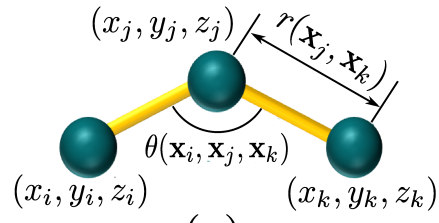

(a)

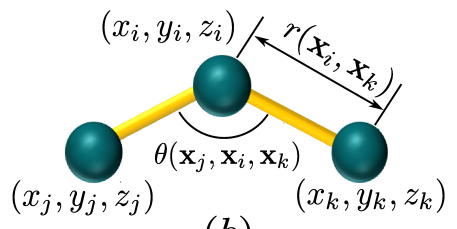

(b)

Fig. 1 Two angle configurations to be distinguished for the calculation of $\left[\mathbf{F}_{j-i-k}^{a}\right]$

\subsection{Modified Morse potential}

The modified Morse potential takes into account the contributions of $E_{\text {stretch }}$ and Engle as

$$
\begin{aligned}
& E_{\text {stretch }}\left(\mathbf{x}_{i}, \mathbf{x}_{j}\right)=D_{e}\left\{\left[1-e^{-\beta\left(r\left(\mathbf{x}_{i}, \mathbf{x}_{j}\right)-r_{0}\right)}\right]^{2}-1\right\} \\
& E_{\text {angle }}\left(\mathbf{x}_{i}, \mathbf{x}_{j}, \mathbf{x}_{k}\right)=\frac{1}{2} K_{\theta}\left(\cos \theta\left(\mathbf{x}_{i}, \mathbf{x}_{j}, \mathbf{x}_{k}\right)-\cos \theta_{0}\right)^{2}
\end{aligned}
$$

where

$$
r\left(\mathbf{x}_{i}, \mathbf{x}_{j}\right)=\sqrt{\left(\mathbf{x}_{i}-\mathbf{x}_{j}\right)^{2}}
$$

and

$$
\cos (\theta)=\frac{\left(\mathbf{x}_{i}-\mathbf{x}_{j}\right) \cdot\left(\mathbf{x}_{k}-\mathbf{x}_{j}\right)}{\sqrt{\left(\mathbf{x}_{i}-\mathbf{x}_{j}\right)^{2}} \sqrt{\left(\mathbf{x}_{k}-\mathbf{x}_{j}\right)^{2}}}
$$

in the case (a) of figure 1 and

$$
\cos (\theta)=\frac{\left(\mathbf{x}_{j}-\mathbf{x}_{i}\right) \cdot\left(\mathbf{x}_{k}-\mathbf{x}_{i}\right)}{\sqrt{\left(\mathbf{x}_{j}-\mathbf{x}_{i}\right)^{2}} \sqrt{\left(\mathbf{x}_{k}-\mathbf{x}_{i}\right)^{2}}}
$$

for the case (b) of figure 1 .

Numerical parameters of this model for graphene and nanotubes are provided in Table 1.

Trigonometric form of $E_{\text {angle }}$ has been adopted, where $E_{\text {angle }}$ is function of $\cos \theta$. This representation does not require computation of an Arccos term or their 


\begin{tabular}{ll}
\hline \hline$r_{0}$ & $1.39 \times 10^{-10} \mathrm{~m}$ \\
$D_{e}$ & $6.03105 \times 10^{-19} \mathrm{Nm}$ \\
$\beta$ & $2.625 \times 10^{10} \mathrm{~m}^{-1}$ \\
$\theta_{0}$ & $2.094 \mathrm{rad}$ \\
$k_{\theta}$ & $0.9 \times 10^{-18} \mathrm{Nm} / \mathrm{rad}^{2}$ \\
\hline \hline
\end{tabular}

Table 1 Parameters for the modified Morse potential [5] in carbon systems.

derivatives, which is computationally much more efficient. Both representations coincide for angles close to $\theta_{0}$, which justifies the choice of the trigonometric one.

To solve the nonlinear equations (7) in an efficient and robust manner, ANM is introduced in the following sections. This is the first time that ANM is applied to deal with nanoscale problems. For reasons of clear illustration, we adopted in this work the modified Morse potential since it offers a convenient and differentiable mathematical form. It should be noted that ANM can also be implemented on potential models such as the Tersoff [40]-Brenner [8] potential. However, these potentials present numerical difficulties since they are discontinuous or present singularities. To overcome these issues, regularization techniques have been developed in the framework of ANM, which initially aimed at problems involving severe nonlinearities, such as contact problems [2,3] and plasticity problems [1]. To facilitate the computing of the asymptotic series coefficients, automatic differentiation toolboxes [20] are also available, allowing us to consider complex but differentiable potential functions. Certainly, most potential functions require appropriate adaptations using the aforementioned regularization techniques, which is the scope of future works. As to the validity of the modified Morse potential compared to the Brenner model, interested readers are invited to check [5].

\section{Asymptotic numerical method}

Asymptotic Numerical Method (ANM) has been proven to be an efficient method to deal with nonlinear problems in fluid and solid mechanics $[2,28,30]$. The principle is to associate a perturbation technique with an appropriate numerical resolution scheme, such as the finite element method. This allows transforming a given nonlinear problem into a sequence of linear problems to be solved successively, leading to a numerical representation of the solution in the form of power series truncated at relatively high orders. Once the series are fully determined, an accurate approximation of the solution path is provided inside a determined validity range. Unlike classical techniques, this method does not require iterative corrections thanks to the high order predictor [2]. As series expansion is needed, application of perturbation techniques requires the problem solution to be sufficiently smooth. That is why for problems involving singularities, regularization procedures must be used. Furthermore, for efficiency concerns, the governing equations are systematically set into quadratic forms, which greatly simplifies the definition of recurrence formulae and reduces computational time. Details of these procedures can be found in $[48,25,47,46,1,19,6,7]$. Recent work [23] extended application of ANM to large scale problems involving several millions of degrees of freedom. 
In this section, we recall the basics of ANM for solving nonlinear equations. Attention is focused on the treatment of severe nonlinearities, which often occur within atomistic systems due to the complex form of the interatomic potentials.

Since nonlinear problems frequently lead to complex functionals for which direct implementation of $\mathrm{ANM}$ is inconvenient, it is strongly recommended that the problem equation be set into quadratic forms before applying the perturbation procedure. For reasons of simplicity, we consider the following nonlinear equation as example:

$$
u^{3}=f
$$

where $u$ is the unknown variable and $f$ a variable source term. This nonlinear form involves a cubic function with respect to the unknown $u$. To obtain an equivalent form of Eq. (16) containing only quadratic functions of $u$, we introduce an additional variable $v$ such that $v=u^{2}$. We then reformulate Eq. (16) as:

$$
\left\{\begin{array}{l}
u v=f \\
u^{2}=v
\end{array} .\right.
$$

The newly introduced $v$ is computed and stored as an intermediate variable, which together with $u$ contributes to generate the recurrent formula of $f$. We solve the problem by expressing $f, u$ and $v$ in the form of high order power series, which are developed with respect to a path parameter " $a "$ :

$$
\left\{\begin{array}{l}
u(a)=u_{0}+a u_{1}+a^{2} u_{2}+\ldots+a^{N} u_{N} \\
v(a)=v_{0}+a v_{1}+a^{2} v_{2}+\ldots+a^{N} v_{N} \\
f(a)=f_{0}+a f_{1}+a^{2} f_{2}+\ldots+a^{N} f_{N}
\end{array}\right.
$$

where $N$ is the truncation order of the series. The key point of the method consists in computing all the power series coefficients: once the series are determined, we reconstruct the problem's solution by evaluating the series Eq. (18) following the parameter " $a$ " which varies from 0 to its limit $a_{\max }$ which refers to the step length. $a_{\max }$ is computed considering that the relative difference between solutions of any two consecutive orders remains sufficiently small [11]. The expression of $a_{\max }$ will be given at the end of this section. By substituting the series expansion Eq. (18) into Eq. (17), we obtain the following linear problems for order 1:

$$
\left\{\begin{array}{l}
u_{0} v_{1}+u_{1} v_{0}=f_{1} \\
2 u_{0} u_{1}=v_{1}
\end{array}\right.
$$

By substituting Eq. (19)-2 into Eq. (19)-1, Eq. (19) is transformed into the tangent problem with respect to $u_{1}$ :

$$
\left\{\begin{array}{l}
3 u_{0}^{3} u_{1}=f_{1} \\
2 u_{0} u_{1}=v_{1}
\end{array}\right.
$$


Similarly for order $p,(2 \leqslant p \leqslant N)$, the linear problem is found to be:

$$
\left\{\begin{array}{l}
u_{0} v_{p}+u_{p} v_{0}+\sum_{r=1}^{p-1} u_{r} v_{p-r}=f_{p} \\
2 u_{0} u_{p}+\sum_{r=1}^{p-1} u_{r} u_{p-r}=v_{p} .
\end{array}\right.
$$

Again, when we substitute Eq. (21)-2 into Eq. (21)-1, and we formulate $f$ at order $p$ as:

$$
3 u_{0}^{2} u_{p}+u_{0} \sum_{r=1}^{p-1} u_{r} u_{p-r}+\sum_{r=1}^{p-1} u_{r} v_{p-r}=f_{p} .
$$

We note by comparing Eq. (20)-1 and Eq. (22), that the same (linear) tangent operator is conserved for all orders, which allows representing Eq. (22) by:

$$
f_{p}=L_{t}\left(u_{p}\right)+f_{p}^{n l},
$$

valid for $1 \leqslant p \leqslant N$, where

$$
L_{t}(.)=3 u_{0}^{2}
$$

and where

$$
f_{p}^{n l}=u_{0} \sum_{r=1}^{p-1} u_{r} u_{p-r}+\sum_{r=1}^{p-1} u_{r} v_{p-r} .
$$

For cases involving more complex problem expressions, for example:

$$
e^{u}=f
$$

we seek its equivalent quadratic form by considering the differential of Eq. (26):

$$
f \mathrm{~d} u=\mathrm{d} f,
$$

which defines the linear problem at order 1 as:

$$
f_{0} u_{1}=f_{1}
$$

and the linear problem at order $p,(2 \leqslant p \leqslant N)$ as

$$
f_{0} u_{p}+\frac{1}{p} \sum_{r=1}^{p-1} f_{r} u_{p-r}=f_{p}
$$

A large class of problems involving more complex nonlinearities can be set into quadratic form. The above framework applies to more general nonlinear differential operators, such as the ones arising from discretized finite element equations of mechanical problems (see the following references for more details $[30,46,2,3,28]$ ).

As the validity range of the series is limited, a continuation technique is used to compute the whole solution branch in a step by step manner. The procedure consists in determining the maximal value of the loading path parameter $a$ by 
imposing that the relative difference between the solutions for two consecutive orders must be small by comparison with an accuracy parameter $\delta$ [11]:

$$
a_{\max }=\left(\delta \frac{\left\|u_{1}\right\|}{\left\|u_{N}\right\|}\right)^{1 /(N-1)} .
$$

where $\|$. $\|$ denotes the euclidean norm. Note that $a_{\max }$ is computed a posteriori by using the series coefficients already obtained from solving the $N$ sets of linear problems. As a consequence, the step length is auto-adaptive and completely automatic, as opposed to classical iterative algorithms. In the next section, we present how this technique can be applied to atomistic simulation.

\section{ANM formulation for atomistic simulations}

Equilibrium configuration is achieved by minimizing the energy of the system. Given that the motion of any atom within the system induces variations of the total energy, seeking the state of minimum energy requires that the internal forces be balanced by external loads. For a given atom $i$, interatomic forces are derived from the total energy of the system, which is calculated taking into account all bonded atom pairs and adjacent atom triples. As presented in section 2.1, the sum of interatomic forces acting on the atom $i$ due to bond stretch is written as:

$$
\mathbf{F}_{k}^{s}=\frac{\partial}{\partial \mathbf{x}_{k}}\left(\sum_{(i, j) \in \Gamma_{1}} E_{\text {stretch }}\left(\mathbf{x}_{i}, \mathbf{x}_{j}\right)\right)
$$

Stretching energy is calculated by considering all covalent bonds within the system, but only those directly connected to the atom $k$ lead to meaningful derivatives, see Fig. 2. Similarly, forces due to bond angle bending are derived from angular bond potential evaluated at all the atom triples, so:

$$
\mathbf{F}_{l}^{a}=\frac{\partial}{\partial \mathbf{x}_{l}}\left(\sum_{(i, j, k) \in \Gamma_{2}} E_{\text {angle }}\left(\mathbf{x}_{i}, \mathbf{x}_{j}, \mathbf{x}_{k}\right)+\sum_{(j, i, k) \in \Gamma_{2}} E_{\text {angle }}\left(\mathbf{x}_{j}, \mathbf{x}_{i}, \mathbf{x}_{k}\right)\right) .
$$

Derivative is zero for angular bonds unrelated to the atom $l$. By taking into account the two angle-configurations presented in Fig. 1, nine angular bonds Fig. 2 contribute to the computation of $\mathbf{F}_{l}^{a}$.

Equilibrium at the atom $i$ requires perfect balance between interatomic force contributions respectively evaluated at three directly connected pair bonds and nine related angular bonds, and possible external loads. Solving the problem defined at atom $i$ consists in applying the procedures presented in section 2 on each of the force contributions.

In the following, we discuss in detail the ANM implementation of the bond stretching force $\mathbf{F}_{i}^{s}$ defined at an atom pair $(i-j)$ and acting on the atom $i$. Its formulation has been presented in section 2.1 and 2.2. By condensing equations Eq. (8) Eq. (11) Eq. (13), we obtain:

$$
\mathbf{F}_{i}^{s}=2 D_{e} \beta \frac{\mathbf{x}_{i}-\mathbf{x}_{j}}{\sqrt{\left(\mathbf{x}_{i}-\mathbf{x}_{j}\right)^{2}}} e^{-\beta\left(\sqrt{\left(\mathbf{x}_{i}-\mathbf{x}_{j}\right)^{2}}-r_{0}\right)}\left[1-e^{-\beta\left(\sqrt{\left(\mathbf{x}_{i}-\mathbf{x}_{j}\right)^{2}}-r_{0}\right)}\right],
$$




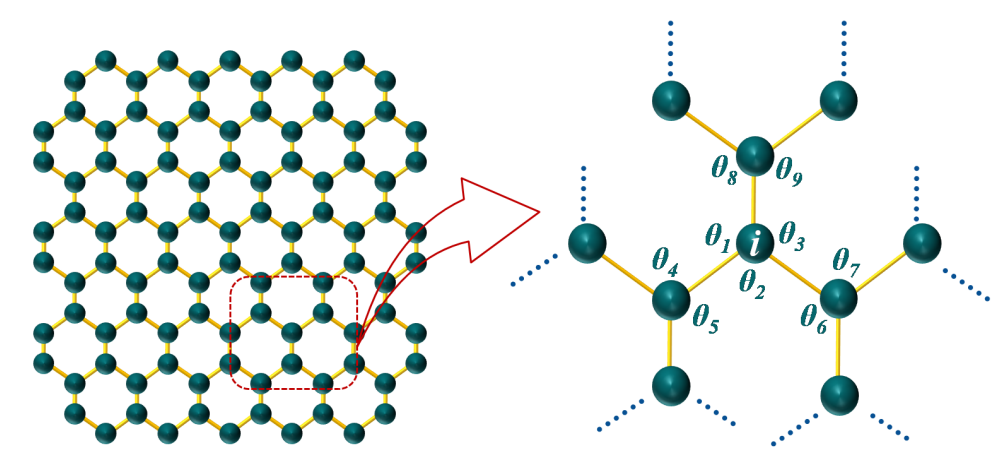

Fig. 2 Calculation of $\mathbf{F}_{i}^{a}$. At each atom i, $\mathbf{F}_{i}^{a}$ corresponds to the sum of angle forces evaluated at angles 1 to 9 . Derivation at other angles of the domain leads to 0 .

where $D_{e}, \beta$ and $r_{0}$ are material constants. Application of ANM requires the problem equation Eq. (33) be written in a convenient form so as to easily deduce the recurrent formulae of the problem. Following the procedure described in section 3, additional intermediate variables are introduced: $\mathbf{u}, r, \alpha, B$ and $\mathbf{A}$, such that

$$
\left\{\begin{array}{l}
\mathbf{u}=\mathbf{x}_{i}-\mathbf{x}_{j} \\
r=\sqrt{\mathbf{u} \cdot \mathbf{u}} \\
\alpha=e^{-\beta\left(r-r_{0}\right)} \\
B=\alpha(1-\alpha) \\
\mathbf{A}=\frac{\mathbf{u}}{\sqrt{\mathbf{u} \cdot \mathbf{u}}}
\end{array}\right.
$$

The given problem Eq. (33) can then be set in quadratic form by applying

$$
\left\{\begin{array}{l}
\mathbf{F}_{i}^{s}=2 D_{e} \beta B \mathbf{A} \\
r \mathbf{A}=\mathbf{u} \\
B=\alpha(1-\alpha) \\
-\beta \alpha \mathrm{d} r=\mathrm{d} \alpha \\
r^{2}=\mathbf{u} \cdot \mathbf{u} \\
\mathbf{u}=\mathbf{x}_{i}-\mathbf{x}_{j}
\end{array} .\right.
$$

All the unknowns including the additional intermediate variables are now expanded into power series with respect to the parameter " $a$ ":

$$
\left\{\begin{array}{l}
\lambda(a)=\lambda_{0}+a^{1} \lambda_{1}+a^{2} \lambda_{2}+\ldots+a^{N} \lambda_{N} \\
\mathbf{u}(a)=\mathbf{u}_{0}+a^{1} \mathbf{u}_{1}+a^{2} \mathbf{u}_{2}+\ldots+a^{N} \mathbf{u}_{N} \\
r(a)=r_{0}+a^{1} r_{1}+a^{2} r_{2}+\ldots+a^{N} r_{N} \\
\alpha(a)=\alpha_{0}+a^{1} \alpha_{1}+a^{2} \alpha_{2}+\ldots+a^{N} \alpha_{N} \\
\mathbf{A}(a)=\mathbf{A}_{0}+a^{1} \mathbf{A}_{1}+a^{2} \mathbf{A}_{2}+\ldots+a^{N} \mathbf{A}_{N} \\
B(a)=B_{0}+a^{1} B_{1}+a^{2} B_{2}+\ldots+a^{N} B_{N}
\end{array}\right.
$$

where $\lambda$ is the external load parameter (Eq. (6)). In the practical implementation, the order of truncation $N$ is generally chosen between 10 and 20 in the interest of 
optimal efficiency. A numerical study has been proposed in [47] that investigated the choice of the optimal truncation order as function of the problem size.

We then substitute the series development Eq. (36) into the system Eq. (35), transforming this nonlinear problem into $N$ linear ones, represented by the following system (for $1 \leqslant k \leqslant N$ ) :

$$
\left\{\begin{array}{l}
\left(\mathbf{F}_{i}^{s}\right)_{k}=2 D_{e} \beta\left(\mathbf{A}_{0} B_{k}+\mathbf{A}_{k} B_{0}\right)+2 D_{e} \beta \sum_{i=1}^{k-1} \mathbf{A}_{i} B_{k-i} \\
\mathbf{A}_{k}=\frac{1}{r_{0}}\left(\mathbf{u}_{k}-\mathbf{A}_{0} r_{k}\right)-\frac{1}{r_{0}} \sum_{i=1}^{k-1} r_{i} \mathbf{A}_{k-i} \\
B_{k}=\alpha_{k}-2 \alpha_{k} \alpha_{0}-\sum_{i=1}^{k-1} \alpha_{i} \alpha_{k-i} \\
\alpha_{k}=-\beta \alpha_{0} r_{k}-\frac{\beta}{k} \sum_{i=1}^{k-1}(k-i) \alpha_{i} r_{k-i} \\
r_{k}=\frac{1}{r_{0}} \mathbf{u}_{k} \cdot \mathbf{u}_{0}+\frac{1}{2 r_{0}} \sum_{i=1}^{k-1} \mathbf{u}_{i} \cdot \mathbf{u}_{k-i}
\end{array}\right.
$$

where each relation contains a summation term depending on the series coefficients that have been calculated at previous orders. In other words, we fully determine the series Eq. (36) once we are given the initial solution at order 0. The last four equations are substituted into the first one, leading to a condensed formula of $\left(\mathbf{F}_{i}^{s}\right)_{k}$ with respect to the displacement vector $\mathbf{u}_{k}$ :

$$
\left(\mathbf{F}_{i}^{s}\right)_{k}=\mathcal{L} s_{0}\left(\mathbf{u}_{k}\right)+\mathbf{F} s_{k}^{n l},
$$

in which $\mathcal{L} s_{0}(\cdot)$ can be identified as a tangent operator only depending on initial solutions and where $\mathbf{F} s_{k}^{n l}$ incorporates solutions previously obtained for orders up to $k-1$, representing the nonlinear contribution within $\left(\mathbf{F}_{i}^{s}\right)_{k}$. The above formulation only takes into account the effect of bond stretch. As for the bond angle bending (force $\left(\mathbf{F}_{i}^{s}\right)_{k}$ ), given the expressions Eq. (14) and Eq. (15), we apply the similar procedure as presented so far and obtain a set of similar recurrent formula (compared to Eq. (37).) for the respective series coefficients, leading to the bond angle bending force $\mathbf{F}_{i}^{a}$, also written as a function of the displacement vector as

$$
\left(\mathbf{F}_{i}^{a}\right)_{k}=\mathcal{L} a_{0}\left(\mathbf{u}_{k}\right)+\mathbf{F} a_{k}^{n l}
$$

To model the global structure incorporating every atomic interaction, the above computing procedures should be repeated on every bonded atom pair and atom triplets contained in the system. This requires evaluating $\mathbf{F}_{i}^{s}, \mathbf{F} s^{n l}, \mathbf{u}$ and $\mathcal{L} s_{0}(\cdot)$ for each covalent pair bond, as well as $\mathbf{F}^{a}, \mathbf{F} a^{n l}, \mathbf{u}$ and $\mathcal{L} a_{0}(\cdot)$ for each atom angular bond, all of which should be assembled into their respective global vector or matrix systems, leading to the global system at order $k$ :

$$
\mathbf{F}_{k}^{i n t}=\mathcal{L}_{0}\left(\mathbf{u}_{k}\right)+\mathbf{F}_{k}^{n l} .
$$


Taking into account the external load, which is conditioned by the prescribed force vector $\mathbf{F}$ and governed by the parameter $\lambda$, equilibrium can be expressed by

$$
\mathcal{L}_{0}\left(\mathbf{u}_{k}\right)=\lambda_{k} \mathbf{F}+\mathbf{F}_{k}^{n l},
$$

where the nonlinear force $\mathbf{F}^{n l}$ equals to 0 for order 1 . Similarly to $\mathcal{L} s_{0}$ and $\mathcal{L} a_{0}$, the global tangent operator $\mathcal{L}_{0}$ fully depends on the initial solutions and is the same for all orders, thus only requires to be evaluated once.

Note that the problem is defined by the equilibrium equation Eq. (41), in which both $\mathbf{u}$ and $\lambda$ are unknowns. Solution of the system Eq. (41) under quasi-static loading conditions are usually obtained by using continuation procedures based on prescribed force, displacement or arc length. For these algorithms, an additional constraint equation [11] is required. In the present work, we propose the following arc-length type continuation condition:

$$
a=\left\langle\mathbf{u}-\mathbf{u}_{0}, \mathbf{u}_{1}\right\rangle+\left(\lambda-\lambda_{0}\right) \lambda_{1},
$$

in which $\langle$,$\rangle indicates scalar product. The equilibrium equation Eq. (41) is solved$ for each order successively by taking into account the above additional equation. For order 1, the nonlinear force $\mathbf{F}^{n l}$ is 0 , and the problem is found as:

$$
\left\{\begin{array}{l}
\mathcal{L}_{0}\left(\mathbf{u}_{1}\right)=\lambda_{1} \mathbf{F} \\
\left\langle\mathbf{u}_{1}, \mathbf{u}_{1}\right\rangle+\lambda_{1}^{2}=1
\end{array} .\right.
$$

In order to solve this system, let's introduce the notation $\hat{\mathbf{u}}$, such that $\mathbf{u}_{1}=$ $\lambda_{1} \hat{\mathbf{u}}$, which allows rewriting Eq. (43)-1 as:

$$
\mathcal{L}_{0}(\hat{\mathbf{u}})=\mathbf{F} .
$$

$\hat{\mathbf{u}}$ is obtained by solving Eq. (44). $\lambda_{1}$ is then computed considering Eq. (43)-2, which leads to:

$$
\lambda_{1}=\frac{ \pm 1}{\sqrt{\langle\hat{\mathbf{u}}, \hat{\mathbf{u}}\rangle+1}} .
$$

Inspired by the continuation procedure provided by the Riks' method, we determine the sign of $\lambda$ by comparing the tangent vector calculated at the end of the previous step with the one obtained at the beginning of the present step $[11,2,27$, $28]$.

For order $k,(2 \leqslant p \leqslant N)$, the problem is found to be:

$$
\left\{\begin{array}{c}
\mathcal{L}_{0}\left(\mathbf{u}_{k}\right)=\lambda_{k} \mathbf{F}+\mathbf{F}_{k}^{n l} \\
\left\langle\mathbf{u}_{k}, \mathbf{u}_{1}\right\rangle+\lambda_{k} \lambda_{1}=0
\end{array} .\right.
$$

We follow the same idea as for order 1 to solve the above system. Here, we introduce the notation $\mathbf{u}_{k}^{n l}$, such that $\mathbf{u}_{k}=\lambda_{k} \hat{\mathbf{u}}+\mathbf{u}_{k}^{n l}$, where $\hat{\mathbf{u}}$ is the solution of Eq. (44) and $\mathbf{u}_{k}^{n l}$ is the solution of the following linear problem:

$$
\mathcal{L}_{0}\left(\mathbf{u}_{k}^{n l}\right)=\mathbf{F}_{k}^{n l} .
$$

$\lambda_{k}$ is then computed by considering Eq. (46)-2, which leads to:

$$
\lambda_{k}=-\lambda_{1}\left\langle\mathbf{u}_{k}^{n l}, \mathbf{u}_{1}\right\rangle \text {. }
$$




\section{Numerical examples}

In this section, three examples are provided to test the accuracy, efficiency and robustness of the method. In order to compare the performances of ANM and Newton-Raphson techniques on a fair basis, both procedures have been implemented through the same programming language with similar code architectures.

\subsection{Extension of a graphene sheet}

In this first test, a graphene sheet is submitted to in-plane traction load. This simple example is selected to show the efficiency of the present approach even in absence of buckling. The model is composed of 306 atoms. Atoms on the lower edge of the structure are fixed, and a force $F$ is uniformly applied on those situated on the opposite edge, as depicted in figure 3. Initial interatomic distance is 1.39 $\AA$.

The main parameters of the ANM algorithm are the truncation order $N$ and the accuracy parameter $\delta$. A large value of $N$ significantly improves the asymptotic step length, thus reduces the number of steps necessary to obtain the whole solution branch. However, this also requires large CPU time for computing the series coefficients. Several works have shown that the optimum value of the truncation order is situated between 10 and 20 for moderately large size problems. For example, Zahrouni et al. [47] showed that for a buckling shell problem involving $10^{4}$ degrees of freedom, the optimum truncation order is about 15 and a similar study in the framework of plasticity analysis showed that $N=10$ seems to be optimum [1]. Recall as well that within ANM procedure, no correction phases are needed thanks to the high order prediction. The accuracy parameter $\delta$ also affects the step length. If $\delta$ is chosen to be very small, large number of steps is necessary to achieve the whole computation but with very high accuracy along the solution branch. A large value of $\delta$ may lead to poor accuracy regarding residuals. The optimum value of $\delta$ is not easy to choose by the user. For this reason and to make the ANM algorithm less dependent on the choice of the accuracy parameter $\delta$, a high order predictor has been associated to a high order corrector in the framework of ANM $[22,19,2,3]$. In the proposed study, we focus our attention on the applicability of ANM to nanostructures, the truncation order for the series and the accuracy parameter are chosen as $N=20$ and $\delta=10^{-4}$.

The displacement evolution is analyzed as function of the external load. A Newton Raphson solution with arc-length control (Riks' method [33,34]) is computed for comparison. Both solutions are presented in figure 4 .

We can note a very good agreement between both solutions. In figure 4, each dot corresponds to a Newton step and circle dots correspond to ANM step ends. As a comparison, the ANM solution was obtained with only 4 steps, corresponding to 4 matrices inversions. The total number of matrices inversions and computational times are compared in Table 2. Note that in a geometric nonlinear framework, NR procedure requires updating the tangent matrix for each corrective iteration, which increases the number of tangent matrix inversion, while the ANM does not require any correction step. The evolution of residual during the simulation is plotted in figure 5. It can be shown that the norm of the residual remains small during all the simulation. 


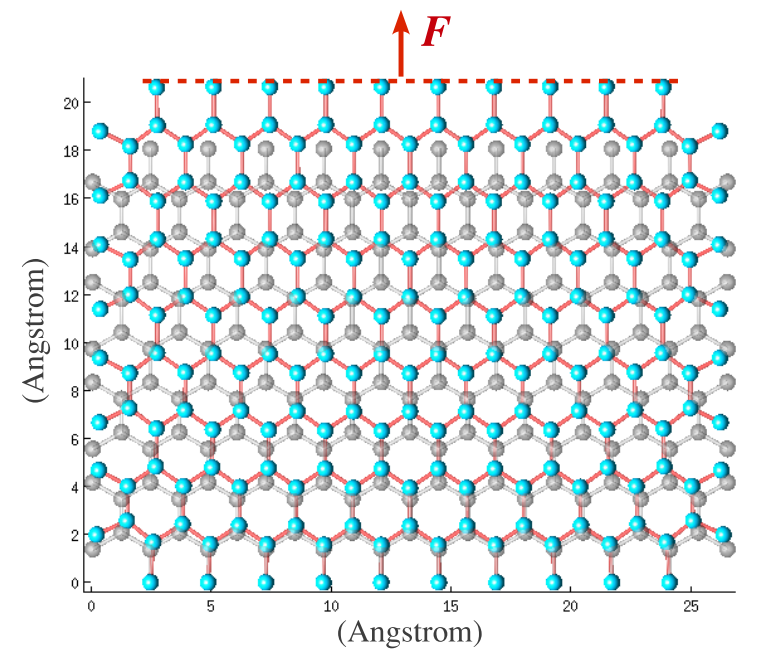

Fig. 3 Extension of graphene subjected to tensile traction force $F$.

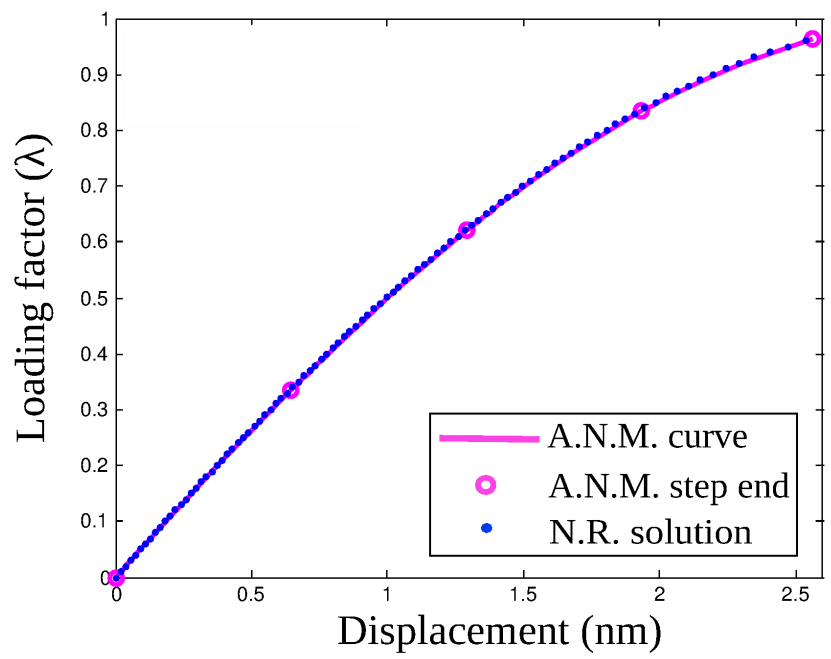

Fig. 4 Comparison betwenn ANM and Newton-Raphson solutions for the tensile traction of the graphene sheet. We note that the ANM solution is found in 4 steps, against 98 for NR.

Both NR and ANM are implemented using the same Matlab code, to provide comparable results regarding computational costs. Room for further improvement is then left regarding the use of a compiled programming language. All computations have been performed on a common PC equipped with a dual-core $2.40 \mathrm{GHz}$ processor and 2 GB memory. 


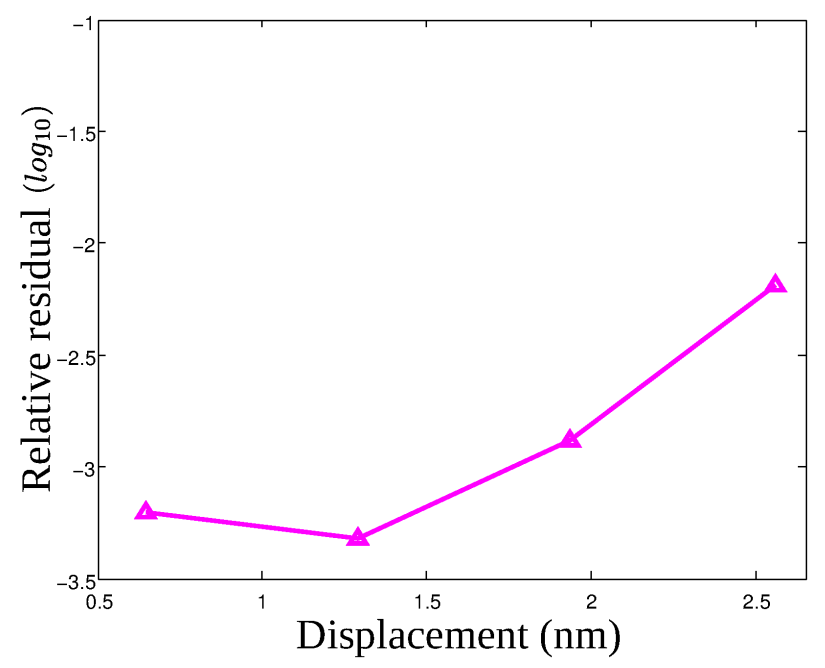

Fig. 5 Accuracy of the ANM results: evolution of the relative residual norm during the simulation for the traction test on a graphene sheet.

\begin{tabular}{ccc}
\hline \hline & NR & ANM \\
\hline Number of step & 98 & 4 \\
Number of tangent matrix inversion & 196 & 4 \\
Computation time & $1 \mathrm{~min} 10 \mathrm{~s}$ & $5.6 \mathrm{~s}$ \\
\hline \hline
\end{tabular}

Table 2 Comparison of computational cost for ANM and NR solutions for the graphene sheet traction example.

\subsection{Buckling of a nanotube}

In this second example, a slender nanotube (NT) is submitted to axial compression, inducing global buckling. We consider a $(10,0)$ zigzag nanotube clamped at one end and subjected to a compression force $F$ uniformly applied on atoms at one free end, where the horizontal displacement is eliminated (see fig 6). The dimensions of the NT are $L=11 \mathrm{~nm}$ and $R=0.2 \mathrm{~nm}$ with the initial interatomic distance chosen as $1.39 \AA$. This example is selected to assess the validity of the method when dealing with problems involving instabilities. The following ANM parameters have been chosen: $N=20$ and $\delta=10^{-5}$.

As in the previous example, we investigate the displacement at the free end of the structure as function of the loading force. To trigger the buckling, a small perturbation compressive radial force $f$ is applied at the middle of the structure. Here, two cases are studied: the first one with $f=F / 100$, and the other with $f=F / 10, F$ being the axial compressive force. Solutions obtained with ANM and $\mathrm{NR}$ are compared in figure 7. An arc-length control method is employed for the NR algorithm, as well as an adaptive step-length strategy to deal with the high nonlinearity involved. 


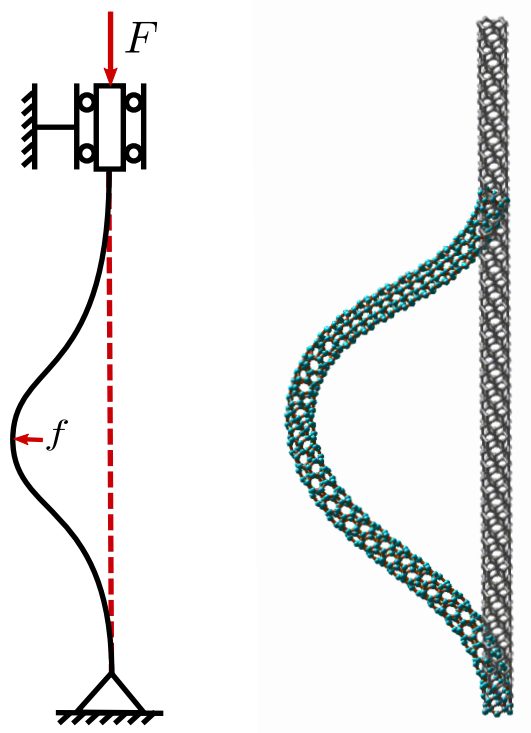

Fig. 6 Compression of nanotube: under force control $F$.

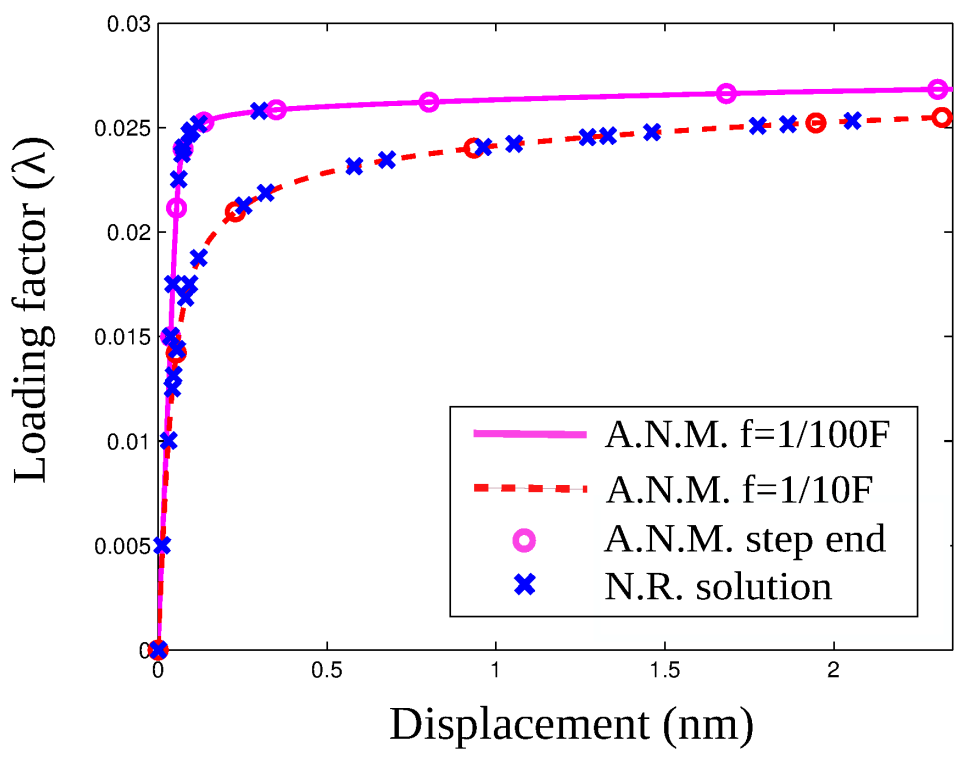

Fig. 7 Buckling of a nanotube subjected to a compressive force $F$.

It can be noted from figure 7 that when the perturbation force is small ( $f=$ $F / 100$ ), the response curve shows stiffer behaviours of the structure compared with the case where $f=F / 10$. A good agreement between NR and ANM can be noticed. However, for the most severe case $(f=F / 100)$, the NR diverges, even though arc-length and adaptive step length strategies have been employed. On the 
other hand, ANM is able to provide the solution for the whole loading range in a few steps. Figure 8 shows the residual norm evolution during the simulation for the ANM solution. A comparison of performance for both methods is provided in Table 3 only for the case where $f=F / 10$, which was completed for both methods.

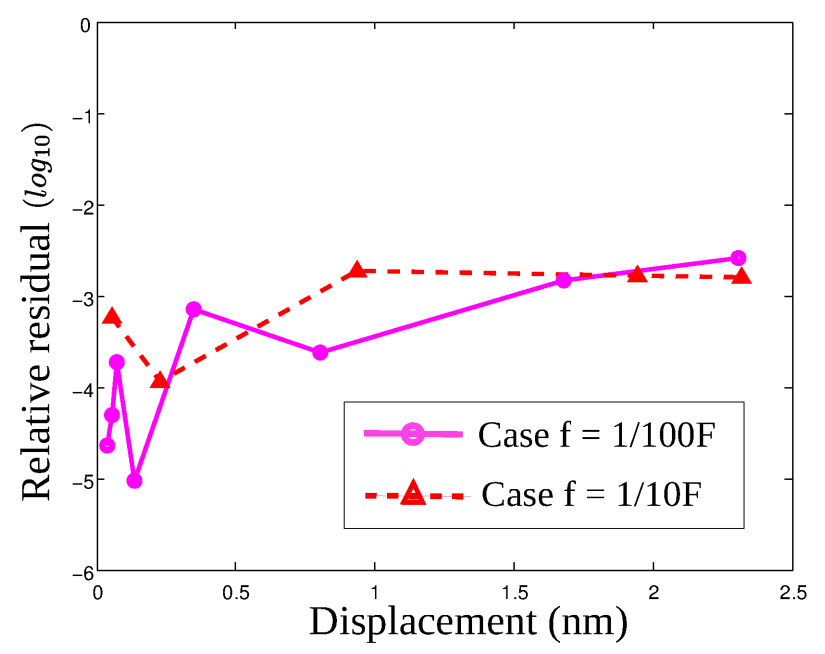

Fig. 8 Evolution of relative residual for each ANM step end. Residual globally remains small, confirming the accuracy of the solution.

\begin{tabular}{ccc}
\hline \hline & NR & ANM \\
\hline Number of steps & 20 & 5 \\
Number of tangent matrix inversions & 50 & 5 \\
Computation time & $4 \min 7 \mathrm{~s}$ & $39 \mathrm{~s}$ \\
\hline \hline
\end{tabular}

Table 3 Comparison of computational costs for NR and ANM methods in the case of the nanotube buckling test, $f=F / 10$.

Beside accuracy and computational efficiency, the present example demonstrates the robustness of ANM for dealing with problems involving non-linearities. Based on high order prediction, the method does not require corrective iterations and avoids convergence issues which represent a challenge for conventional methods.

\subsection{Radial compression of a nanotube}

In this last example, we considered a $(18,0)$ zigzag nanotube clamped at both ends as shown in figure 9 . The dimensions of the NT are $L=9.5 \mathrm{~nm}, R=0.35 \mathrm{~nm}$ and 
the initial interatomic distance is $1.39 \AA$. A horizontal displacement is prescribed on one of the atoms situated in the middle of the structure. As the structure is clamped at both ends, the loading conditions simply induce extension to all atomic bonds within the structure but those around the loading area, where local buckling occur. ANM parameters are chosen as $N=10$ and $\delta=10^{-4}$. Results are provided in figure 10.
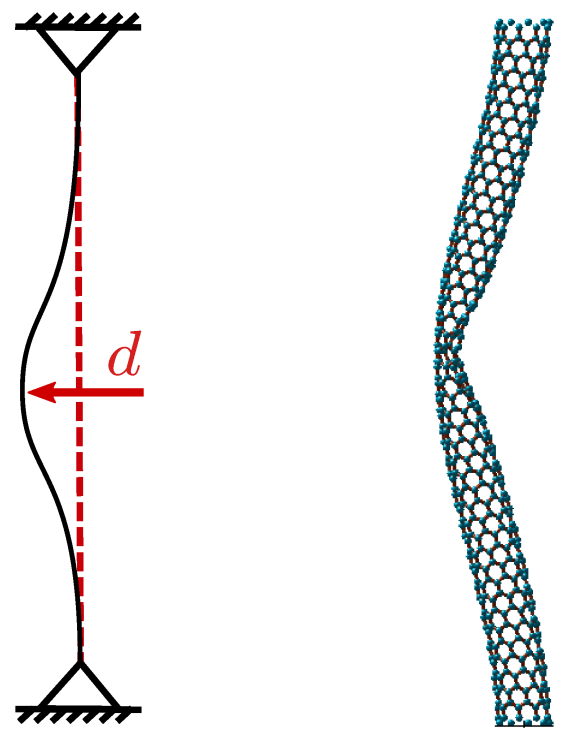

Fig. 9 Local deformation of a nanotube.

At the beginning of the load, many instabilities locally occur near the compressive loading point. This yields a transitional zone where fine oscillations can be observed (see the zooming figure 11). This phenomenon is challenging for both methods. In the case of ANM, it induces small step lengths in the transition region. We recall that in the context of ANM, the length of each step is automatically determined according to Eq. (30). In the case of the Newton method, very small steps are required to guarantee the convergence. However, the NR was not able to converge for the whole loading range. On the other hand, the ANM was able to complete the simulation in 29 steps, to be compared with 49 for the NR, corresponding to 100 matrices inversion, only for the region where the solution can be computed. In the region where both simulations could be achieved, excellent agreement is observed between both methods. This test demonstrates the high robustness and efficiency of the proposed method.

\section{Conclusion}

A new method has been proposed to compute the quasi-static response of atomistic structures with possible instabilities. The technique extends for the first time a perturbation method called Asymptotic Numerical Method (ANM) to atomistic 


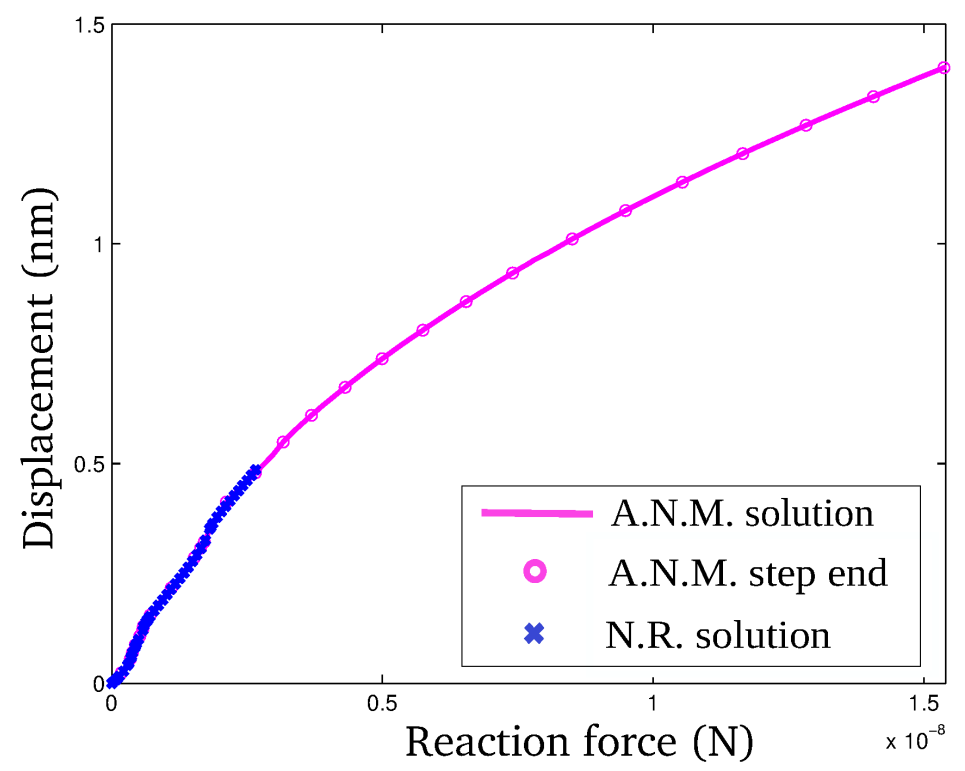

Fig. 10 Comparison between ANM and Newton solutions in the case of local compression of a nanotube. A first transition zone is observed, where local instabilities occur. The NR method diverges and is not able to provide the solution for the whole simulation.

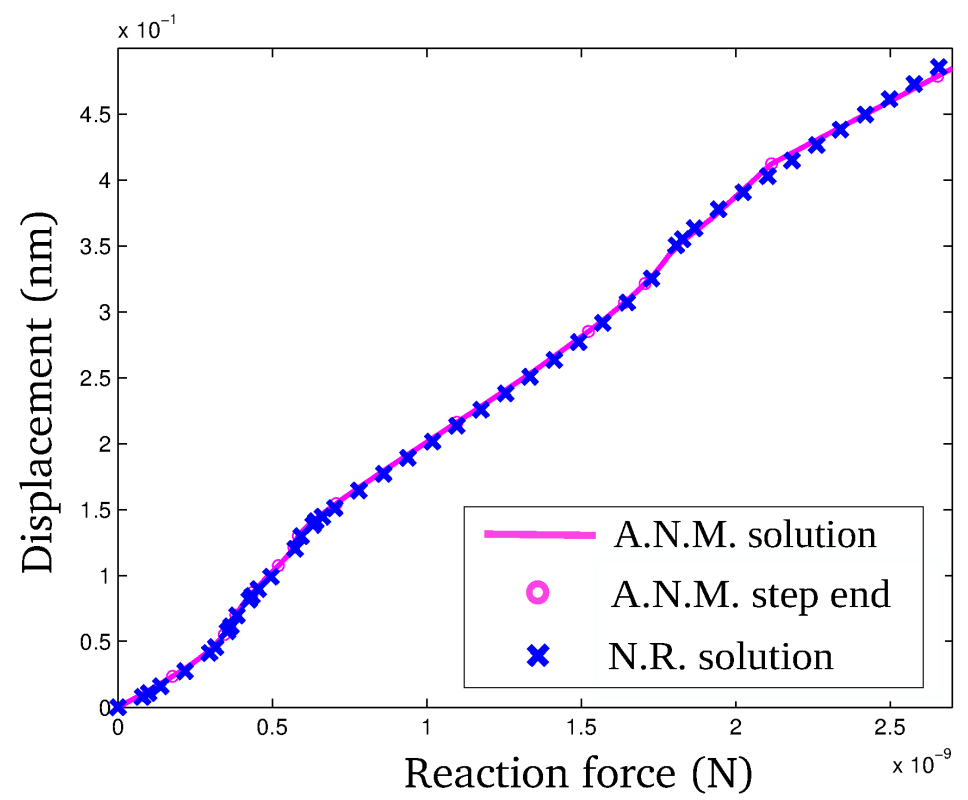

Fig. 11 Detail of the transition region: comparison between NR and ANM solutions.

simulations. In that context, a continuous representation of the atomic displacements with respect to the load evolution can be obtained, by means of a high order 
series expansion. Introducing this expansion into the set of nonlinear equations related to minimizing the potential energy results in a series of linear problems which have to be solved successively to provide the different terms of the series. Large loading steps can then be obtained, as opposed to first-order methods like Newton-Raphson procedures. Furthermore, issues related to limit points and instabilities are naturally avoided in this context. The results presented in this work, involving thin carbon structures like graphene and nanotubes, demonstrate the high efficiency and robustness of the present approach, as compared to Newton Raphson method. In the most severe cases involving global or local buckling, the NR solution diverged, even using an arc-length control and adaptive step length procedures, while the ANM was able to provide the solution for the whole loading range. In the case where no instability occurs, the ANM provides the whole solution within a few steps only. An interesting extension of this work consists in applying an automatic differentiation procedure $[18,10]$, which provides all formulations and series coefficients in an automatic manner. Efforts in this direction have been initiated and will be proposed in a forthcoming study.

\section{References}

1. Abichou, H., Zahrouni, H., Potier-Ferry, M.: Asymptotic numerical method for problems coupling several nonlinearities. Computer Methods in Applied Mechanics and Engineering (191), 5795-5810 (2002)

2. Aggoune, W., Zahrouni, H., Potier-Ferry, M.: High-order prediction-correction algorithms for unilateral contact problems. Journal of Computational and Applied Mathematics 168(1-2), 1-9 (2004)

3. Aggoune, W., Zahrouni, H., Potier-Ferry, M.: Asymptotic numerical methods for unilateral contact. International Journal for Numerical Methods in Engineering 68, 605-631 (2006)

4. Arroyo, M., Belytschko, T.: Nonlinear mechanical response and rippling of thick multiwalled carbon nanotubes. Physical Review Letters 91, 215,505 (2003)

5. Belytschko, T., Xiao, S.P., Schatz, G.C., Ruoff, R.S.: Atomistic simulations of nanotube fracture. Physics Review B 65(235430) (2002)

6. Boutyour, E.H., Zahrouni, H., Potier-Ferry, M., Boudi, M.: A.N.M. for buckling analysis of shell structures with large rotations. Journal of Computational and Applied Mathematics (168), 77-85 (2004)

7. Boutyour, E.H., Zahrouni, H., Potier-Ferry, M., Boudi, M.: Bifurcation points and bifurcated branches by an asymptotic numerical method and Padé approximants. International Journal for Numerical Methods in Engineering (60), 1987-2012 (2004)

8. Brenner, D.W.: Empirical potential for hydrocarbons for use in simulating the chemical vapor deposition of diamond films. Physical Review B 42, 9458-9471 (1990)

9. Chandra, Y., R.Chowdhury, S.Adhikari, F.Scarpa: Elastic instability of bilayer graphene using atomistic finite element. Physica E 44, 12-16 (2011)

10. Charpentier, I., Potier-Ferry, M.: Différentiation automatique de la méthode asymptotique numérique typée : l'approche diamant. Comptes Rendus Mécanique 336, 336-340 (2008)

11. Cochelin, B.: A path-following technique via an asymptotic-numerical method. Computers and Structures 53(5), 1181-1192 (1994)

12. Davidovitcha, B., Schrolla, R.D., Vellab, D., Adda-Bediab, M., Cerdad, E.A.: Prototypical model for tensional wrinkling in thin sheets. Proceedings of the National Acadamy of Sciences of the United States of America 108(45), 18,227-18,232 (2011)

13. Fletcher, R., Powell, M.J.D.: A rapidly convergent descent method for minimization. The Computer Journal 6(2), 163-168 (1963)

14. Fletcher, R., Reeves, C.M.: Function minimization by conjugate gradients. The Computer Journal 7(2), 149-154 (1964)

15. Giannopoulos, G.I.: Elastic buckling and flexural rigidity of graphene nanoribbons by using a unique translational spring element per interatomic interaction. Computational Materials Science 53(1), 388-395 (2012) 
16. Govindjee, S., Sackman, J.L.: On the use of continuum mechanics to estimate the properties of nanotubes. Solid State Communications 110(4), 227-230 (1999)

17. Jiang, C., Singamaneni, S., Merrick, E., Tsukruk, V.V.: Complex buckling instability patterns of nanomembranes with encapsulated gold nanoparticle arrays. Nano Letters 6(10), 2254-2259 (2006)

18. Koutsawa, Y., Charpentier, I., Daya, E.M., Cherkaoui, M.: A generic approach for the solution of nonlinear residual equations. part i: The diamant toolbox. Computer Methods in Applied Mechanics and Engineering 198, 572-577 (2008)

19. Lahmam, H., Cadou, J.M., Zahrouni, H., Damil, N., Potier-Ferry, M.: High order predictorcorrector algorithms. International Journal for Numerical Methods in Engineering (55), 685-704 (2002)

20. Lejeune, A., Béchet, F., Boudaoud, H., Mathieu, N., Potier-Ferry, M.: Object-oriented design to automate a high order non-linear solver based on asymptotic numerical method. Advances in Engineering Software 48, 70-88 (2012)

21. Lu, Q., Bhattacharya, B.: The role of atomistic simulations in probing the small-scale aspects of fracture-: a case study on a single-walled carbon nanotube. Engineering Fracture Mechanics 72(13), 2037-2071 (2005)

22. Mallil, E., Lahmam, H., Damil, N., Potier-Ferry, M.: An iterative process based on homotopy and perturbation techniques. Computer Methods in Applied Mechanics and Engineering 190(13-14)

23. Medale, M., Cochelin, B.: A parallel computer implementation of the asymptotic numerical method to study thermal convection instabilities. Journal of Computational Physics 22(228)

24. Morse, P.M.: Diatomic molecules according to the wave mechanics. ii. vibrational levels. Physics Review 34, 57-64 (1929)

25. Najah, A., Cochelin, B., Damil, N., Potier-Ferry, M.: A critical review of asymptotic numerical method. Archieves of Computational Methods in Engineering 5, 3-22 (1998)

26. Neek-Amal, M., Peeters, F.: Buckled circular monolayer graphene: a graphene nano-bowl. Journal of Physics: Condensed Matter 23(045002) (2011)

27. Nezamabadi, S., Yvonnet, J., Zahrouni, H., Potier-Ferry, M.: A multilevel computational strategy for handling microscopic and macroscopic instabilities. Computer Methods in Applied Mechanics and Engineering 198, 2099-2110 (2009)

28. Nezamabadi, S., Zahrouni, H., Yvonnet, J.: Solving hyperelastic material problems by asymptotic numerical method. Computational Mechanics 47(1), 77-92 (2011)

29. Nezamabadi, S., Zahrouni, H., Yvonnet, J., Potier-Ferry, M.: A multiscale finite element approach for buckling analysis of elastoplastic long fibre composites. International Journal for Multiscale Computational Engineering 8(3), 287-301 (2010)

30. Potier-Ferry, M., Damil, N., Braikat, B., Descamps, J., Cadou, J.M., Cao, H.L., Hussein, A.E.: Traitement des fortes non-linéarités par la méthode asymptotique numérique. Comptes Rendus de l'Académie des Sciences - Series IIB - Mechanics-Physics-ChemistryAstronomy 324(3), 171-177 (1997)

31. Quinn, D.D., Wilber, J.P., Clemons, C.B., Young, G.W., Buldum, A.: Buckling instabilities in coupled nano-layers. International Journal of Non-linear Mechanics 42(4), 681-689 (2007)

32. Rapaport, D.C.: The art of molecular dynamics simulation. Cambridge University Press, Cambridge (2004)

33. Riks, E.: The application of Newton's method to the problem of elastic stability. Journal of Applied Mechanics 39, 1060-1065 (1972)

34. Riks, E.: An incremental approach to the solution of snapping and buckling problems. International Journal of Solids and Structures 15, 529-551 (1979)

35. Ru, C.Q.: Column buckling of multiwalled carbon nanotubes with interlayer radial displacements. Physics Review B 62(24), 16,962 (2000)

36. Ru, C.Q.: Effect of van der waals forces on axial buckling of a double-walled carbon nanotube. Journal of Applied Physics 87(10), 7227 (2000)

37. Ru, C.Q.: Axially compressed buckling of a double-walled carbon nanotube embedded in an elastic medium. Journal of the Mechanics and Physics of Solids 49, 1265-1279 (2001)

38. Ru, C.Q.: Degraded axial buckling strain of multiwalled carbon nanotubes due to interlayer slips. Journal of Applied Physics 89(6), 3426 (2001)

39. Schroll, R.D., Katifori, E., Davidovitch, B.: Elastic building blocks for confined sheets. Physics Review Letters 106, 074,301 (2011). DOI 10.1103/PhysRevLett.106.074301. URL http://link.aps.org/doi/10.1103/PhysRevLett.106.074301 
40. Tersoff, J.: New empirical approach for the structure and energy of covalent systems Physical Review B 37, 6991-7000 (1988)

41. Vandeparre, H., Piñeirua, M., Brau, F., Roman, B., Bico, J., Gay, C. Bao, W., Lau, C.N., Reis, P.M., Damman, P.: Wrinkling hierarchy in constrained thin sheets from suspended graphene to curtains. Physics Review Letters 106, 224,301 (2011). DOI 10.1103/PhysRevLett.106.224301. URL http://link.aps.org/doi/10.1103/PhysRevLett.106.224301

42. Wang, C.Y., Ru, C.Q., Mioduchowski, A.: Elastic buckling of multi-walled carbon nanotubes under high pressure. Journal of Nanoscience and Nanotechnology 3(15), 199-208 (2003)

43. Wanga, Q., Liewb, K., Duana, W.: Modeling of the mechanical instability of carbon nanotubes. Carbon 46, 285-290 (2008)

44. Yakobson, B.I., Brabec, C.J., Bernholc, J.: Nanomechanics of carbon tubes: Instability beyond linear response. Physics Review Letters 76, 2511-2514 (1996)

45. Yap, H.W., Lakes, R.S., Carpick, R.W.: Mechanical instabilities of individual multiwalled carbon nanotubes under cyclic axial compression. Nano Letters 7(5), 1149-1154 (2007)

46. Zahrouni, H., Aggoune, W., Brunelot, J., Potier-Ferry, M.: Asymptotic numerical method for strong nonlinearities. Revue Européenne des Eléments Finis 13(1-2), 97-118 (2004)

47. Zahrouni, H., Cochelin, B., Potier-Ferry, M.: Computing finite rotations by an asymptotic numerical method. Computer Methods in Applied Mechanics and Engineering (175), 71-85 (1999)

48. Zahrouni, H., Potier-Ferry, M., Elasmar, H., Damil, N.: Asymptotic numerical method for nonlinear constitutive laws. Revue européenne des éléments finis 7(7), 841-869 (1998)

49. Zheng, Q., Geng, Y., S.Wang, Z. Li, J.K.K.: Effects of functional groups on the mechanical and wrinkling properties of graphene sheets. Carbon 48, 4315-4322 (2010) 